\title{
Kohn Anomalies and Electron-Phonon Interaction in Graphite
}

\author{
S. Piscanec ${ }^{1}$, M. Lazzeri ${ }^{2}$, Francesco Mauri ${ }^{2}$, A. C. Ferrari ${ }^{1}$, and J. Robertson ${ }^{1}$ \\ ${ }^{1}$ Cambridge University, Engineering Department, Trumpington Street, Cambridge CB2 1PZ, UK \\ ${ }^{2}$ Laboratoire de Minéralogie-Cristallographie de Paris, 4 Place Jussieu, 75252, Paris cedex 05, France
}

(Dated: February 2, 2008)

\begin{abstract}
We demonstrate that graphite phonon dispersions have two Kohn anomalies at the $\boldsymbol{\Gamma}-\mathrm{E}_{2 g}$ and $\mathbf{K}-\mathrm{A}_{1}^{\prime}$ modes. The anomalies are revealed by two sharp kinks. By an exact analytic derivation, we show that the slope of these kinks is proportional to the square of the electron-phonon coupling (EPC). Thus, we can directly measure the EPC from the experimental dispersions. The $\boldsymbol{\Gamma}-\mathrm{E}_{2 g}$ and $\mathbf{K}-\mathrm{A}_{1}^{\prime}$ EPCs are particularly large, whilst they are negligible for all the other modes at $\boldsymbol{\Gamma}$ and $\mathbf{K}$.

PACS numbers: 63.20.Dj, 63.20.Kr, 71.15.Mb, 78.30.-j
\end{abstract}

Carbon nanotubes (CNTs) are at the core of nanotechnology research. They are prototype one dimensional conductors. Metallic nanotubes are predicted to be onedimensional quantum wires with ballistic electron transport [1]. However, high field electrical transport measurements show that the electron-phonon scattering by optical phonons at $\mathbf{K}$ and $\boldsymbol{\Gamma}$ breaks down the ballistic behavior 2]. Electron phonon coupling (EPC) is thus the fundamental bottleneck for ballistic transport. Raman spectroscopy is a most used characterization technique to identify CNTs in terms of their size and electronic properties [3]. The optical phonons at $\mathbf{K}$ and $\boldsymbol{\Gamma}$ are the phonons responsible for the Raman D and G peaks in carbons [4]. The frequency and the intensity of the Raman modes are determined by the EPC matrix elements [5]. The determination of the EPCs is necessary to settle the 35 years debate on the nature of the Raman D peak in carbons [4, 5, 6, 7, 8, 9, 10, 11, 12]. Finally, although graphite phonon dispersions have been widely studied, several contrasting theoretical dispersions were proposed [6, 7, 8, 9, 10, 13, 14]. In particular, the origin of the large overbending of the $\mathbf{K}-\mathrm{A}_{1}^{\prime}$ branch is not yet understood and is associated to an intense EPC [4, 9, 10]. In principle, the electronic and vibrational properties of CNTs can be described by folding the electronic and phonon dispersions of graphite. The precise determination of the graphite EPCs is thus the crucial step to understand the properties of any carbon based material and CNTs in particular. It is then surprising that, despite the vast literature on carbon materials and CNTs, no experimental determination or first principle calculations of the graphite EPCs has been done so far, to the best of our knowledge.

Here we show that in graphite the EPC matrix elements at $\boldsymbol{\Gamma}$ and $\mathbf{K}$ can be directly extracted from the phonon dispersions. We demonstrate two remarkable Kohn anomalies in the phonon dispersions at $\boldsymbol{\Gamma}$ and $\mathbf{K}$, by an exact analytic derivation and accurate density functional theory (DFT) calculations. We prove that the slope of the anomalies is proportional to the EPC square.

A key feature of graphite is the semi-metallic character of the electronic structure. In general, the atomic vibra- tions are partially screened by electrons. In a metal this screening can change rapidly for vibrations associated to certain q points of the Brillouin Zone (BZ), entirely determined by the shape of the Fermi surface. The consequent anomalous behavior of the phonon dispersion is called Kohn anomaly [15]. Kohn anomalies may occur only for wavevectors $\mathbf{q}$ such that there are two electronic states $\mathbf{k}_{1}$ and $\mathbf{k}_{2}=\mathbf{k}_{1}+\mathbf{q}$ both on the Fermi surface 15. The electronic bands dispersions of graphite are, essentially, described by those of an isolated graphene sheet. In graphene, the gap between occupied and empty electronic states is zero only at the two equivalent BZ points $\mathbf{K}$ and $\mathbf{K}^{\prime}$. Since $\mathbf{K}^{\prime}=2 \mathbf{K}$, the two equivalent $\mathbf{K}$ points are connected by the vector $\mathbf{K}$. Thus, Kohn anomalies can occur for $\mathbf{q}=\boldsymbol{\Gamma}$ or $\mathbf{q}=\mathbf{K}$.

We perform calculations within the generalized gradient approximation (GGA) 16], using the density functional perturbation theory (DFPT) scheme [17], which allows the exact (within DFT) computation of phonon frequencies at any BZ point. We use the plane-waves (90 Ry cut-off) and pseudopotential [18] approach. The electronic levels are occupied with a finite fictitious electronic temperature $\sigma$ 19]. This smears out the discontinuities present in the Fermi distribution for $\sigma=0$, and the exact result is recovered for $\sigma \rightarrow 0$. The experimental lattice $\left(a_{\exp }=2.46 \AA, c=6.708 \AA\right)$ is used for graphite, while for graphene we consider both the graphite $a_{\exp }$ and the theoretical values $\left(a_{\mathrm{th}}=2.479 \AA\right)$. Graphene layers are separated by $7.4 \AA$ of vacuum.

Fig. [1]compares the measured optical branches [9] with our calculations at $\sigma=0.02$ Ry. Phonon frequencies are computed exactly for a series of points along the high symmetry lines $\mathbf{\Gamma}-\mathbf{K}$ and $\mathbf{\Gamma}-\mathbf{M}$ and then interpolated with a spline. The agreement with experiments is $\sim 2 \%$, which is the expected accuracy of DFT-GGA. At $\boldsymbol{\Gamma}$ the experimental dispersion is very well reproduced by the calculations with $a_{\text {exp }}$. At $\mathbf{K}$ the upper branch is better described by the calculations with $a_{\mathrm{th}}$.

The most striking feature of these dispersions is the discontinuity in the frequency derivative of the highest optical branches (HOB) at $\boldsymbol{\Gamma}$ and at $\mathbf{K}\left(\mathrm{E}_{2 g}\right.$ and $\mathrm{A}_{1}^{\prime}$ modes). Indeed, near $\boldsymbol{\Gamma}, \hbar \omega_{\mathbf{q}}=\alpha_{\boldsymbol{\Gamma}} q+\hbar \omega_{\boldsymbol{\Gamma}}+\mathcal{O}\left(q^{2}\right), \omega_{\mathbf{q}}$ 


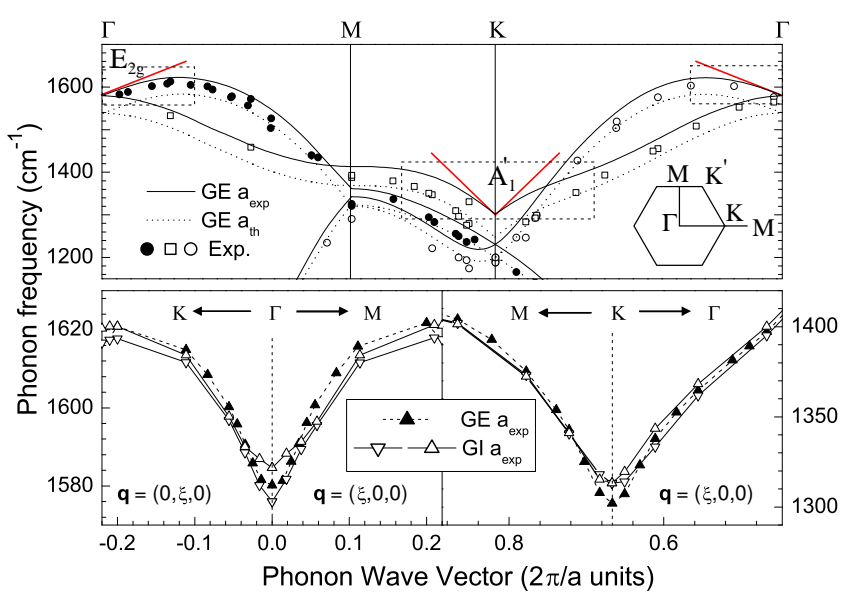

FIG. 1: (Color online) Upper panel: Lines are the phonon dispersion of graphene (GE), calculated at the experimental and equilibrium lattice spacings ( $a_{\exp }$ and $\left.a_{t h}\right)$. Experimental data from Ref. [9]. The red straight lines at $\boldsymbol{\Gamma}$ and $\mathbf{K}$ are obtained from Eqs. 810] The two lower panels correspond to the dotted window in the upper panel. Here, graphite (GI) computed frequencies are also shown. The points are theoretical frequencies obtained by direct calculation. A single GE band corresponds to two almost-degenerate GI-bands.

being the phonon frequency of the HOB at the $\mathbf{q}$ wavevector. Similarly, near $\mathbf{K}, \hbar \omega_{\mathbf{K}+\mathbf{q}^{\prime}}=\alpha_{\mathbf{K}} q^{\prime}+\hbar \omega_{\mathbf{K}}+\mathcal{O}\left(q^{\prime 2}\right)$. Such dependencies cannot be described by a finite set of interatomic force constants, or by a set decaying exponentially with the real-space distance. In these cases the dynamical matrix dependence on $\mathbf{q}$ would be analytic, and, because of symmetry, the highest optical branch near $\boldsymbol{\Gamma}$ and $\mathbf{K}$ would have a flat slope $\left(\alpha_{\boldsymbol{\Gamma}}=\alpha_{\mathbf{K}}=0\right)$. Thus, a non zero $\alpha_{\boldsymbol{\Gamma}}$ or $\alpha_{\mathbf{K}}$ indicates a non-analytic behavior of the phonon dispersion, due to a polynomial decay of the force constants in real space. This explains why it is impossible for any of the often used few-nearest-neighbors force constants approaches to properly describe the HOB phonons near $\mathbf{K}$ and $\boldsymbol{\Gamma}[6,6,8,10,13,14]$. The graphite HOB are almost indistinguishable from those of graphene, Fig. 11 The non-analytic behavior at $\boldsymbol{\Gamma}$ and $\mathbf{K}$ is also present in graphite. At $\boldsymbol{\Gamma}$ the HOB is doubly degenerate, consisting of in-plane anti-phase $\mathrm{E}_{2 \mathrm{~g}}$ movements. Near $\boldsymbol{\Gamma}$ the two modes split in a upper longitudinal optical (LO) branch and a lower transverse optical (TO) branch. $\alpha_{\boldsymbol{\Gamma}} \neq 0$ only for the LO branch.

Fig. 2 plots the HOB as a function of $\sigma$, to clarify the nature of the discontinuities. The results for $\sigma=0.01 \mathrm{Ry}$ and $\sigma=0.02$ Ry are similar, indicating that, on the scale of the figure, the $\sigma \rightarrow 0$ limit is reached. Increasing $\sigma$, the non-analytic behavior is smoothed out. Particularly striking is the behavior around $\mathbf{K}$, where for $\sigma>0.20 \mathrm{Ry}$ the dispersion is almost flat. Within DFPT, the smearing $\sigma$ affects virtual transitions between occupied and empty states, differing in energy by $\lesssim \sigma$. Thus, the smoothing of Fig. 2 indicates that the HOB discontinuities are

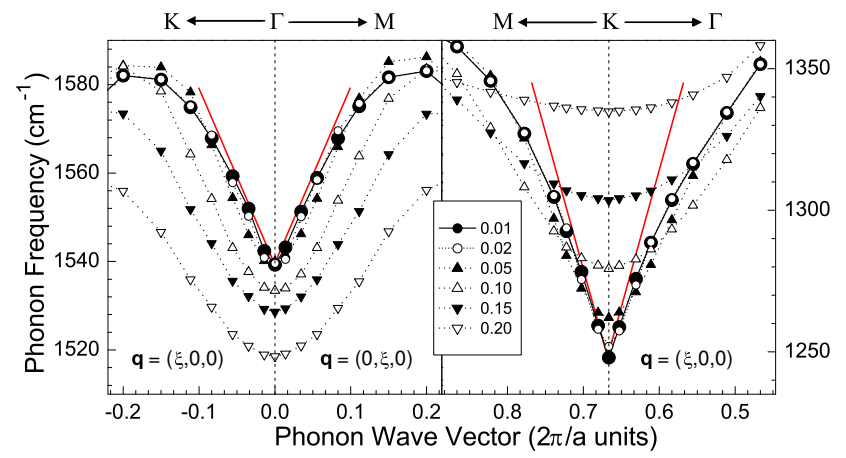

FIG. 2: (Color online) Graphene HOB around $\boldsymbol{\Gamma}$ and $\mathbf{K}$ as a function of smearing. Points are calculated frequencies for $a_{\mathrm{th}}$. Lines guides to the eye. The red straight lines plot Eqs. 810

Kohn anomalies [15], since they are related to an anomalous screening of the electrons around the Fermi energy.

We compute the EPC matrix elements, to understand why the Kohn anomalies affect only the HOB and not the others. For a given phonon mode at the reciprocalspace point $\mathbf{q}$, we call $\Delta V_{\mathbf{q}}$ and $\Delta n_{\mathbf{q}}$ the derivative of the Kohn-Sham potential and of the charge density, with respect to a displacement along the normal coordinate of the phonon. We define:

$$
g_{(\mathbf{k}+\mathbf{q}) i, \mathbf{k} j}=\left\langle\mathbf{k}+\mathbf{q}, i\left|\Delta V_{\mathbf{q}}\left[\Delta n_{\mathbf{q}}\right]\right| \mathbf{k}, j\right\rangle \sqrt{\hbar /\left(2 M \omega_{\mathbf{q}}\right)},
$$

where we consider explicitly the dependence of $\Delta V_{\mathbf{q}}$ on $\Delta n_{\mathbf{q}}$, as indicated by $\Delta V_{\mathbf{q}}\left[\Delta n_{\mathbf{q}}\right] . \quad|\mathbf{k}, i\rangle$ is the electronic Bloch eigen-state with wavevector $\mathbf{k}$, band index $i$, and eigen-energy $\epsilon_{\mathbf{k}, i}$. $M$ is the atomic mass, and $g$ is an energy. The dimensionless EPC [20] is $\lambda_{\mathbf{q}}=2 \sum_{\mathbf{k}, i, j}\left|g_{(\mathbf{k}+\mathbf{q}) i, \mathbf{k} j}\right|^{2} \delta\left(\epsilon_{\mathbf{k}+\mathbf{q}, i}-\epsilon_{\mathrm{F}}\right) \delta\left(\epsilon_{\mathbf{k}, j}-\right.$ $\left.\epsilon_{\mathrm{F}}\right) /\left(\hbar \omega_{\mathbf{q}} N_{\mathrm{F}} N_{\mathrm{k}}\right)$, where $\sum_{\mathbf{k}}$ is a sum on $N_{\mathrm{k}}$ BZ vectors, $N_{\mathrm{F}}$ is the density of states per spin at the Fermi energy $\epsilon_{\mathrm{F}}$. In graphene the Fermi surface is a point, $N_{\mathrm{F}}$ is zero and $\lambda_{\mathbf{q}}$ is not well-defined. Thus, we evaluate [20]:

$$
\frac{2\left\langle g_{\mathbf{q}}^{2}\right\rangle_{\mathrm{F}}}{\hbar \omega_{\mathbf{q}}}=\frac{\lambda_{\mathbf{q}} N_{\mathrm{F}}}{J_{\mathbf{q}}}
$$

where $\langle\ldots\rangle_{\mathrm{F}}$ indicates the average on the Fermi surface of $\left|g_{(\mathbf{k}+\mathbf{q}) i, \mathbf{k} j}\right|^{2}$, and $J_{\mathbf{q}}=1 / N_{k} \sum_{i, j, \mathbf{k}} \delta\left(\epsilon_{\mathbf{k}+\mathbf{q}, i}-\epsilon_{\mathrm{F}}\right) \delta\left(\epsilon_{\mathbf{k}, j}-\right.$ $\left.\epsilon_{\mathrm{F}}\right)$. In graphene, $\left\langle g_{\mathbf{K}}^{2}\right\rangle_{\mathrm{F}}=\sum_{i, j}^{\pi}\left|g_{(2 \mathbf{K}) i, \mathbf{K} j}\right|^{2} / 4$, and $\left\langle g_{\boldsymbol{\Gamma}}^{2}\right\rangle_{\mathrm{F}}=\sum_{i, j}^{\pi}\left|g_{\mathbf{K} i, \mathbf{K} j}\right|^{2} / 4$, where the sums are performed on the two degenerate $\pi$ bands at $\epsilon_{\mathrm{F}}$. For the HOB, we obtain $\left\langle g_{\boldsymbol{\Gamma}}^{2}\right\rangle_{\mathrm{F}}=0.0405 \mathrm{eV}^{2}$ [21] , and $\left\langle g_{\mathbf{K}}^{2}\right\rangle_{\mathrm{F}}=0.0994 \mathrm{eV}^{2}$, corresponding to $2\left\langle g_{\mathbf{q}}^{2}\right\rangle_{\mathrm{F}} /\left(\hbar \omega_{\mathbf{q}}\right)$ of $0.41 \mathrm{eV}$ and $1.23 \mathrm{eV}$ at $\boldsymbol{\Gamma}$ and $\mathbf{K}$, respectively. $2\left\langle g_{\mathbf{K}}^{2}\right\rangle_{\mathbf{F}} /\left(\hbar \omega_{\mathbf{K}}\right)$ is much smaller $(0.02 \mathrm{eV})$ for the doubly degenerate $1200 \mathrm{~cm}^{-1}$ phonon at $\mathbf{K}$ and zero for all the other phonons at $\mathbf{K}$ and at $\boldsymbol{\Gamma}$, consistent with the absence of Kohn anomalies for all these branches. The EPCs values for the graphene HOB are very large in absolute terms. They are comparable to the $39 \mathrm{~K}$ superconductor $\mathrm{MgB}_{2}$, for which 
$\lambda_{\mathbf{q}} N_{\mathrm{F}} / J_{\mathbf{q}}=1.6 \mathrm{eV}[22]$ for each of the two stronglycoupled $\mathrm{E}_{2 \mathrm{~g}}$ branches at $\mathrm{A}$. On the contrary, in alkaline doped $\mathrm{C}_{60}$, the largest $2\left\langle g_{\mathbf{q}}^{2}\right\rangle_{\mathrm{F}} /\left(\hbar \omega_{\mathbf{q}}\right)$ for individual phonon modes are much smaller $(0.02-0.034 \mathrm{eV}$ [20]).

We consider the expression of $\omega_{\mathbf{q}}$ according to perturbation theory [17] (within DFT), to understand the absence of a Kohn anomaly for the $\mathrm{TO} \mathrm{E}_{2 \mathrm{~g}}$ phonon at $\boldsymbol{\Gamma}$ and to correlate the constants $\alpha_{\boldsymbol{\Gamma}}$ and $\alpha_{\mathbf{K}}$ with the magnitude of the EPC. $\omega_{\mathbf{q}}=\sqrt{D_{\mathbf{q}} / M}$, where $D_{\mathbf{q}}$ is the dynamical matrix projected on the phonon normal coordinate:

$$
\begin{aligned}
D_{\mathbf{q}}= & \frac{4}{N_{\mathbf{k}}} \sum_{\mathbf{k}, o, e} \frac{\left|\left\langle\mathbf{k}+\mathbf{q}, e\left|\Delta V_{\mathbf{q}}\left[\Delta n_{\mathbf{q}}\right]\right| \mathbf{k}, o\right\rangle\right|^{2}}{\epsilon_{\mathbf{k}, o}-\epsilon_{\mathbf{k}+\mathbf{q}, e}} \\
& -\int \Delta n_{\mathbf{q}}^{*}(\boldsymbol{r}) K\left(\boldsymbol{r}, \boldsymbol{r}^{\prime}\right) \Delta n_{\mathbf{q}}\left(\boldsymbol{r}^{\prime}\right) d^{3} r d^{3} r^{\prime} \\
& +\int n(\boldsymbol{r}) \Delta^{2} V^{b}(\boldsymbol{r}) d^{3} r .
\end{aligned}
$$

Here a factor 2 accounts for spin degeneracy, $\sum_{o, e}$ is a sum on occupied and empty states, $n(\boldsymbol{r})$ is the charge density, $K\left(\boldsymbol{r}, \boldsymbol{r}^{\prime}\right)=\delta^{2} E_{H x c}[n] /\left(\delta n(\boldsymbol{r}) \delta n\left(\boldsymbol{r}^{\prime}\right)\right), E_{H x c}[n]$ is the Hartree and exchange-correlation functional, and $\Delta^{2} V^{b}$ is the second derivative of the bare (purely ionic) potential. From previous considerations,

$$
\begin{aligned}
\alpha_{\boldsymbol{\Gamma}} & =\hbar \lim _{\mathbf{q} \rightarrow 0} \frac{\omega_{\mathbf{q}}-\omega_{\boldsymbol{\Gamma}}}{q}=\hbar \lim _{\mathbf{q} \rightarrow 0} \frac{D_{\mathbf{q}}-D_{\boldsymbol{\Gamma}}}{2 M \omega_{\boldsymbol{\Gamma}} q}, \\
\alpha_{\mathbf{K}} & =\hbar \lim _{\mathbf{q}^{\prime} \rightarrow 0} \frac{\omega_{\mathbf{K}+\mathbf{q}^{\prime}}-\omega_{\mathbf{K}}}{q^{\prime}}=\hbar \lim _{\mathbf{q}^{\prime} \rightarrow 0} \frac{D_{\mathbf{K}+\mathbf{q}^{\prime}}-D_{\mathbf{K}}}{2 M \omega_{\mathbf{K}} q^{\prime}} .
\end{aligned}
$$

If the dependence of $D_{\mathbf{q}}$ on $\mathbf{q}$ were analytic over all the BZ, $\alpha_{\boldsymbol{\Gamma}}=\alpha_{\mathbf{K}}=0$ (E.g. $D_{\mathbf{q}}-D_{\boldsymbol{\Gamma}}=\mathcal{O}\left(q^{2}\right)$ ). For $\mathbf{q} \neq \boldsymbol{\Gamma}$ and $\mathbf{K}$, the energy denominators in the sum of Eq. 2 are finite and $D_{\mathbf{q}}$ is analytic. The energy denominators go to zero for $\mathbf{q}=\boldsymbol{\Gamma}$ (when $\mathbf{k}=\mathbf{K}$ or $\mathbf{k}=2 \mathbf{K}$ ) and for $\mathbf{q}=\mathbf{K}$ (when $\mathbf{k}=\mathbf{K}$ ), when $o$ and $e$ correspond to the $\pi$ and $\pi^{*}$ bands near the Fermi energy. Due to these singularities, the dynamical matrix is non-analytic for $\mathbf{q}=\mathbf{K}, \boldsymbol{\Gamma}$, thus $\alpha_{\boldsymbol{\Gamma}}$ and $\alpha_{\mathbf{K}}$ can be different from zero. To compute $\alpha_{\mathbf{q}}$, we can replace in Eqs. 34 the full dynamical matrix $D_{\mathbf{q}}$ with its non-analytic component $\tilde{D}_{\mathbf{q}}$, which includes only the sum of Eq. 2 restricted to the $\pi$ electronic bands in an arbitrarily-small but finite circle of radius $k_{\mathrm{m}}$ around the Fermi surface at the $\mathbf{K}$ and/or $2 \mathbf{K}$ points [23].

For $\mathbf{q}$ near $\boldsymbol{\Gamma}$, using the definition of Eq. 1]

$$
\tilde{D}_{\mathbf{q}}=\frac{8 \sqrt{3} M \omega_{\boldsymbol{\Gamma}}}{\hbar} \int_{k^{\prime}<k_{\mathrm{m}}} d^{2} k^{\prime} \frac{\left|g_{\left(\mathbf{K}+\mathbf{k}^{\prime}+\mathbf{q}\right) \pi^{*},\left(\mathbf{K}+\mathbf{k}^{\prime}\right) \pi}\right|^{2}}{\epsilon_{\mathbf{K}+\mathbf{k}^{\prime}, \pi}-\epsilon_{\mathbf{K}+\mathbf{k}^{\prime}+\mathbf{q}, \pi^{*}}} .
$$

Here we have used the substitution $1 / N_{\mathrm{k}} \sum_{\mathrm{k}}=$ $\sqrt{3} / 2 \int d^{2} k^{\prime}$ (the graphene BZ area is $2 / \sqrt{3}$ ), $\mathbf{q}$ and $\mathbf{k}$ points are in units of $2 \pi / a, a$ being the lattice spacing. $\mathbf{k}^{\prime}=\mathbf{k}-\mathbf{K}$. A factor 2 accounts for the contribution of the two equivalent Fermi points. For small $\mathbf{q}$ and $\mathbf{k}^{\prime}$, the EPC matrix elements in the numerator in Eq. 5 are:

$$
\left|g_{\left(\mathbf{K}+\mathbf{k}^{\prime}+\mathbf{q}\right) \pi^{*},\left(\mathbf{K}+\mathbf{k}^{\prime}\right) \pi}^{\frac{\mathrm{LO}}{\mathrm{TO}}}\right|^{2}=\left\langle g_{\mathbf{\Gamma}}^{2}\right\rangle_{\mathrm{F}}\left[1 \pm \cos \left(\theta+\theta^{\prime}\right)\right],
$$

where $\theta$ is the angle between $\mathbf{k}^{\prime}$ and $\mathbf{q}$, and $\theta^{\prime}$ is the angle between $\mathbf{k}^{\prime}+\mathbf{q}$ and $\mathbf{q}$ [24]. The + or $-\operatorname{sign}$ refers to the LO and the TO modes, respectively. In graphene, the electronic bands near the Fermi level have a conic shape. Therefore, for small $\mathbf{q}$ and $\mathbf{k}^{\prime}$,

$$
\epsilon_{\mathbf{K}+\mathbf{k}^{\prime}, \pi}-\epsilon_{\mathbf{K}+\mathbf{k}^{\prime}+\mathbf{q}, \pi^{*}}=-\beta k^{\prime}-\beta\left|\mathbf{k}^{\prime}+\mathbf{q}\right|,
$$

where $\beta=14.1 \mathrm{eV}$ is the slope of the $\pi$ bands within GGA. Replacing Eq. 56 and 7 in Eq. 3] one obtains:

$$
\begin{aligned}
& \alpha_{\boldsymbol{\Gamma}}^{\frac{\mathrm{LO}}{\mathrm{TO}}}=\frac{4 \sqrt{3}\left\langle g_{\boldsymbol{\Gamma}}^{2}\right\rangle_{\mathrm{F}}}{\beta} \times \\
& \lim _{\mathbf{q} \rightarrow 0} \frac{1}{q} \int_{k^{\prime}<k_{\mathrm{m}}} d^{2} k^{\prime}\left[\frac{1 \pm \cos 2 \theta}{2 k^{\prime}}-\frac{1 \pm \cos \left(\theta+\theta^{\prime}\right)}{k^{\prime}+\left|\mathbf{k}^{\prime}+\mathbf{q}\right|}\right] \\
& =\frac{4 \sqrt{3}\left\langle g_{\mathbf{\Gamma}}^{2}\right\rangle_{\mathrm{F}}}{\beta} \int_{0}^{\infty} d y \int_{0}^{2 \pi} d \theta\left[\frac{1}{2}-\frac{y \pm y \cos \left(\theta+\theta^{\prime}\right)}{y+\sqrt{1+y^{2}+2 y \cos \theta}}\right],
\end{aligned}
$$

where $y=k / q$, and $\theta^{\prime}=\arctan [y \sin \theta /(1+y \cos \theta)]$. The integral is zero for the TO mode and $\pi^{2} / 4$ for the LO mode. Thus, as expected, $\alpha_{\boldsymbol{\Gamma}}^{\mathrm{TO}}=0$ and

$$
\alpha_{\boldsymbol{\Gamma}}^{\mathrm{LO}}=\frac{\left\langle g_{\boldsymbol{\Gamma}}^{2}\right\rangle_{\mathrm{F}}}{\beta} \sqrt{3} \pi^{2}=397 \mathrm{~cm}^{-1}
$$

For the $\mathbf{K}$ point, we use an analogous procedure, for transitions from points in the neighborhood of $\mathbf{K}$ to points in the neighborhood of $2 \mathbf{K}$. The EPC matrix elements are:

$$
\left|g_{\left(2 \mathbf{K}+\mathbf{k}^{\prime}+\mathbf{q}^{\prime}\right) \pi^{*},\left(\mathbf{K}+\mathbf{k}^{\prime}\right) \pi}\right|^{2}=\left\langle g_{\mathbf{K}}^{2}\right\rangle_{\mathrm{F}}\left(1+\cos \theta^{\prime \prime}\right),
$$

with $\theta^{\prime \prime}$ the angle between $\mathbf{k}^{\prime}$ and $\mathbf{k}^{\prime}+\mathbf{q}^{\prime}$ [24]. Eq. 4] becomes:

$$
\alpha_{\mathbf{K}}=\frac{2 \sqrt{3}\left\langle g_{\mathbf{K}}^{2}\right\rangle_{\mathrm{F}}}{\beta} \lim _{\mathbf{q}^{\prime} \rightarrow 0} \frac{1}{q^{\prime}} \int_{k^{\prime}<k_{\mathrm{m}}} d^{2} k^{\prime}\left[\frac{1}{k^{\prime}}-\frac{1+\cos \theta^{\prime \prime}}{k^{\prime}+\left|\mathbf{k}^{\prime}+\mathbf{q}^{\prime}\right|}\right] .
$$

The limit of the integral is $\pi^{2} / 2$, and therefore

$$
\alpha_{\mathbf{K}}=\frac{\left\langle g_{\mathbf{K}}^{2}\right\rangle_{\mathrm{F}}}{\beta} \sqrt{3} \pi^{2}=973 \mathrm{~cm}^{-1} \text {. }
$$

The resulting linear dispersions are plotted in Fig. 11 and Fig. 2 As expected, the phonon slopes near the discontinuities are very well reproduced. Finally, we note that, within a first-neighbors tight-binding (TB) approximation, the EPCs at $\boldsymbol{\Gamma}$ and $\mathbf{K}$ are not independent, since:

$$
\frac{\left\langle g_{\mathbf{K}}^{2}\right\rangle_{\mathrm{F}} \omega_{\mathbf{K}}}{\left\langle g_{\mathbf{\Gamma}}^{2}\right\rangle_{\mathrm{F}} \omega_{\Gamma}}=2
$$

The validity of this relation is supported by our DFT result, for which $\left(\left\langle g_{\mathbf{K}}^{2}\right\rangle_{\mathrm{F}} \omega_{\mathbf{K}}\right) /\left(\left\langle g_{\boldsymbol{\Gamma}}^{2}\right\rangle_{\mathrm{F}} \omega_{\boldsymbol{\Gamma}}\right)=2.02$.

Eqs. 810] allow us to directly measure the EPCs at $\Gamma$ 
and $\mathbf{K}$ from the experimental phonon slopes. The phonon branches around $\boldsymbol{\Gamma}$ have been determined by several groups with a close agreement of the measured data 13]. The fit for $q \leq 0.15$ of the measurements in Fig. [1 9] with $\hbar \omega_{\mathbf{q}}=\hbar \omega_{\boldsymbol{\Gamma}}+\alpha_{\boldsymbol{\Gamma}}^{\mathrm{LO}} q+\gamma q^{2}$, gives $\alpha_{\boldsymbol{\Gamma}}^{\mathrm{LO}}=340 \mathrm{~cm}^{-1}$, and $\left\langle g_{\Gamma}^{2}\right\rangle_{\mathrm{F}}=0.035 \mathrm{eV}^{2}$ [21]. The available data around $\mathbf{K}$ are much more scattered. However, from Eq. 11 we get $\left\langle g_{\mathbf{K}}^{2}\right\rangle_{\mathrm{F}}=0.086 \mathrm{eV}^{2}$. These values are in excellent agreement with our calculations and validate our results.

The EPCs near $\mathbf{K}$ between $\pi^{*}$ bands [25] allow the accurate determination of the intensity and shape of the Raman D peak. This will be the topic of future publications. Here, we remark that the $\mathrm{A}_{1}^{\prime}$ branch has the biggest $\left\langle g_{\mathbf{K}}^{2}\right\rangle_{\mathrm{F}}$ amongst $\mathbf{K}$ phonons. Thus, the $\mathrm{D}$ peak is due to the highest optical branch starting from the $\mathbf{K}-\mathrm{A}_{1}^{\prime}$ mode [4, 9, 10, 11], not to the branch starting from the $\mathbf{K}-\mathrm{E}^{\prime}$ mode, as in [5, 6, 7, 8, 12]. Also, the D peak shifts linearly with laser excitation $\operatorname{energy}\left(\sim 50 \mathrm{~cm}^{-1} / \mathrm{eV}[12]\right)$. This is at odds with the flat slope of the $\mathbf{K}-\mathrm{A}_{1}^{\prime}$ branch obtained by previous calculations [6, 7, 8, 10, 13, 14]. But it is consistent with the $\mathbf{K}-\mathrm{A}_{1}^{\prime}$ linear-dispersion we get. The D peak dispersion reflects the slope of the Kohn anomaly at $\mathbf{K}$ and provides another independent measure of the EPCs. From ref. [5], for $q^{\prime} \rightarrow 0$, the D peak dispersion is $\sim \alpha_{\mathbf{K}} / \beta$. This gives $\left\langle g_{\mathbf{K}}^{2}\right\rangle_{\mathrm{F}} \sim 0.072 \mathrm{eV}^{2}$ and, from Eq. 11] $\left\langle g_{\Gamma}^{2}\right\rangle_{\mathrm{F}} \sim 0.029 \mathrm{eV}^{2}$. The EPCs derived in this way are a lower limit since the experimental D peak dispersion is measured with laser excitation energies $\geq 1 \mathrm{eV}$ [2]. This corresponds to the phonon slope at $q^{\prime} \geq 0.035$, which, from Fig. 2 underestimates by $30 \%$ the slope at $q^{\prime}=0$. Taking this into account, the EPCs independently inferred from the $\mathrm{D}$ peak dispersion are as well in excellent agreement with our calculations.

Due to the reduced dimensionality, we predict even stronger Kohn anomalies for metallic CNTs, and no anomaly for semiconducting CNTs. This is the key to differentiate the electrical nature of CNTs by Raman spectroscopy. A softening of CNT phonons corresponding to the graphene $\boldsymbol{\Gamma}-\mathrm{E}_{2 g}$ mode was recently reported [14]. We expect a stronger softening for the phonons corresponding to the graphene $\mathbf{K}-\mathrm{A}_{1}^{\prime}$ mode, since $\left.\left\langle g_{\mathbf{K}}^{2}\right\rangle_{\mathrm{F}}\right\rangle\left\langle g_{\boldsymbol{\Gamma}}^{2}\right\rangle_{\mathrm{F}}$.

In conclusion, we have demonstrated the presence of two remarkable Kohn anomalies in the phonon dispersions of graphite, revealed by two kinks for the $\boldsymbol{\Gamma}-\mathrm{E}_{2 g}$ and $\mathbf{K}-\mathrm{A}_{1}^{\prime}$ modes. Even if Kohn anomalies have been observed in many materials [17], graphite is the first real material where a simple analytic description of the anomaly is possible. Indeed, we proved, by an exact analytic derivation, that the slope of the kinks is proportional to the ratio between square EPC matrix elements and the slope of the $\pi$ bands at the Fermi energy. As a consequence, we directly derived the EPCs at $\boldsymbol{\Gamma}$ and $\mathbf{K}$ from the experimental phonon dispersions. The values we obtain are in excellent agreement with calculations. Finally, our $\left\langle g_{\Gamma}^{2}\right\rangle_{\mathrm{F}}$ and $\left\langle g_{\mathbf{K}}^{2}\right\rangle_{\mathrm{F}}$ values, with Eqs. 6]9 and 25], can be used to determine the mean free path for electron scattering by optical phonons. This gives the limit of ballistic transport in CNTs and is of great scientific and technologic importance 2]. This calculation can be done, within the folding model, using the Fermi golden rule, and will be reported elsewhere.

We thank C. Brouder, M. Calandra and S. Reich for useful discussions. Calculations were performed at HPCF (Cambridge) and IDRIS (Orsay) using PWSCF [26]. S.P. was supported by the EU project FAMOUS and the Marie Curie Fellowship IHP-HPMT-CT-2000-00209. A.C.F. acknowledges funding from the Royal Society.

[1] C. T. White, T. N. Todorov, Nature 393, 240 (1998)

[2] Z. Yao et al. Phys. Rev. Lett. 84, 2941 (2000); A. Javey et al. Phys. Rev. Lett. 92, 106804 (2004).

[3] A.M. Rao et al. Science 275, 187 (1997).

[4] A.C. Ferrari and J. Robertson Phys. Rev. B 61, 14095 (2000); ibid. 64, 075414 (2001).

[5] C. Thomsen, S. Reich Phys. Rev. Lett. 85, 5214 (2000).

[6] M.J. Matthews et al. Phys. Rev. B 59, 6585 (1999).

[7] A. Grüneis et al. Phys. Rev. B 65, 155405 (2002).

[8] R. Saito et al. Phys. Rev. Lett. 88, 027401 (2002).

[9] J. Maultzsch et al. Phys. Rev. Lett. 92, 075501 (2004).

[10] C. Mapelli et al. Phys. Rev. B 60, 12710 (1999). C. Castiglioni et al. Synth. Met.139, 885 (2003).

[11] F. Tuinstra, J.Koenig J. Chem. Phys. 53, 1126 (1970).

[12] I.Pocsik et al. J. Non-Cryst. Solids 227-230, 1083 (1998).

[13] G. Kresse et al. Europhys. Lett. 32, 729 (1995); P. Pavone et al. Physica B 219-220, 439 (1996); for a review: L. Wirtz and A. Rubio Solid. State Comm. to appear.

[14] O. Dubay, G. Kresse Phys. Rev. B 67, 035401 (2003).

[15] W. Kohn Phys. Rev. Lett. 2, 393 (1959).

[16] J.P. Perdew et al. Phys. Rev. Lett. 77, 3865 (1996).

[17] S. Baroni et al. Rev. Mod. Phys. 73, 515 (2001).

[18] N. Troullier, J.L. Martins Phys. Rev. B 43, 1993 (1991).

[19] M. Methfessel and A.T. Paxton Phys. Rev. B 40, 3616 (1989). We used an Hermite-Gauss smearing of order 1. For graphene we use a $32 \times 32 \times 1 \mathrm{BZ}$ grid for $\sigma \geq 0.02 \mathrm{Ry}$, and a $64 \times 64 \times 1$ for $\sigma=0.01 \mathrm{Ry}$. For graphite we use $32 \times 32 \times 4$ with $\sigma=0.02$ Ry. With these parameters, the frequencies converge to less than $1 \mathrm{~cm}^{-1}$.

[20] O. Gunnarsson Rev. Mod. Phys. 69, 575 (1997).

[21] A much smaller value for $\left\langle g_{\Gamma}^{2}\right\rangle_{\mathrm{F}}\left(0.003 \mathrm{eV}^{2}\right)$ has been obtained with a tight-binding model in J. Jiang et al., Chem. Phys. Lett. 392, 383 (2004), see Fig. 2.

[22] A. Shukla et al., Phys. Rev. Lett. 90, 095506 (2003); at the A point $J_{\mathbf{q}}=0.625(\mathrm{eV})^{-2}$.

[23] $\Delta n_{\mathbf{q}}$ is also non-analytic in $\mathbf{q}$. E.g. at $\boldsymbol{\Gamma}$, at the lowest order, $\Delta n_{\mathbf{q}}-\Delta n_{\boldsymbol{\Gamma}}$ is proportional to $q$. However, the non-analyticity of $\Delta n_{\mathbf{q}}$ gives a contribution to $D_{\mathbf{q}}-D_{\boldsymbol{\Gamma}}$ of order $q^{2}$, since Eq. 2 is stationary with respect to $\Delta n_{\mathbf{q}}$.

[24] This angular dependence was derived using a simple tight-binding model and reproduces very accurately the behavior of our fully ab-initio calculations.

[25] For the branch near $\mathrm{A}_{1}^{\prime}$ at $\mathbf{K},\left|g_{\left(2 \mathbf{K}+\mathbf{k}^{\prime}+\mathbf{q}^{\prime}\right) \pi^{*},\left(\mathbf{K}+\mathbf{k}^{\prime}\right) \pi^{*}}\right|^{2}=$ $\left\langle g_{\mathbf{K}}^{2}\right\rangle_{\mathrm{F}}\left(1-\cos \theta^{\prime \prime}\right)$. For the branches near the $\mathrm{E}_{2 g}$ at $\boldsymbol{\Gamma}$ $\left|g_{\left(\mathbf{K}+\mathbf{k}^{\prime}+\mathbf{q}\right) \pi^{*},\left(\mathbf{K}+\mathbf{k}^{\prime}\right) \pi^{*}}^{\mathrm{LO} / \mathrm{TO}}\right|^{2}=\left\langle g_{\mathbf{\Gamma}}^{2}\right\rangle_{\mathrm{F}}\left[1 \mp \cos \left(\theta+\theta^{\prime}\right)\right]$. 
[26] S. Baroni et al. http://www.pwscf.org 